\title{
A TORSION THEORY FOR ABELIAN CATEGORIES
}

\author{
BY \\ SPENCER E. DICKSON
}

1. Introduction and preliminaries. This paper deals with a generalization of the concept of torsion Abelian groups. Our purpose is twofold. On the one hand, we treat the concept of torsion axiomatically so as to avoid certain pathology which may be associated with definitions too closely linked with a particular Abelian category (cf. [7] and [10]). On the other hand, we establish a procedure by means of which a canonical torsion theory can be singled out for any given Abelian category $\mathscr{C}$ furnished with enough structure for the existence of certain restricted infinite sums and products, and investigate the possibility of a primary decomposition theorem for that canonical torsion theory.

In $\$ 2$ some useful characterizations of torsion theories are given. The concept of torsion theory is equivalent to the notion of idempotent radical in the sense of Maranda [11], and affords some new characterizations of these. The concept of torsion theory is closely associated with the concept of strongly complete Serre classes discussed in [6], [8], [1], [13], and [15]. The essential difference is that the Serre class is closed under taking subobjects, while the class of torsion objects associated with a torsion theory is not in general closed under taking subobjects.

In $\S 3$ we consider operators $L$ and $R$, defined on classes of objects of $\mathscr{C}$ which lead to the two closure operators $T$ and $F$. The latter operators, when applied to subclasses of the objects of $\mathscr{C}$ yield torsion classes, or torsion-free classes, respectively.

In $\$ 4$ we go about the task of constructing the canonical torsion theory and obtain an approximation to a primary decomposition theorem. Counterexamples show that this approximation is best-possible in the sense that the primary decomposition does not hold in an arbitrary Abelian category.

Finally in the last section we give a necessary and sufficient condition for the primary decomposition to be valid in any subcomplete Abelian category having injective envelopes. This primary decomposition is always valid for modules over a commutative Noetherian ring.

The author wishes to express his gratitude to his thesis adviser E. A. Walker for his inspiration and guidance and to New Mexico State University for the esprit de corps of its mathematics section. The first four sections of this paper are for the most part included in the author's doctoral dissertation.

Presented to the Society, January 23, 1964; received by the editors April 15, 1964. 
We shall assume throughout this paper unless otherwise specified $\left({ }^{1}\right)$ that $\mathscr{C}$ is a subcomplete Abelian category in the sense below such that for any object $A$, there is a set-indexed family $\mathscr{S}(A)$ of nonequivalent monomorphisms to $A$ such that any monomorphism to $A$ is equivalent to some member of $\mathscr{S}(A)$. Further we call a member of $\mathscr{S}(A)$ a subobject of $A$, and identify it with its domain if no confusion arises. With this convention, if $a: A \rightarrow B$ is in $\mathscr{S}(B)$, we sometimes call $A$ the subobject and $a$ the inclusion, or canonical injection, and write $A \subseteq B$. In this case we denote the object Coker $a$ by $B / A$. We call the category $\mathscr{C}$ subcomplete, if for any family $\left\{A_{u} \mid u \in U\right\}$ of subobjects of a fixed object $A$, the infinite sum $\sum_{u \in U} A_{u}$ and the infinite product $\prod_{u \in U}\left(A / A_{u}\right)$ exist in $\mathscr{C}$. The following proposition is then easily verified, for the subcomplete Abelian category $\mathscr{C}$.

Proposition 1.1. For any object $A$ of $\mathscr{C}, \mathscr{S}(A)$ is a complete lattice under the following operations: For a subfamily $\left\{A_{u} \mid u \in U\right\}$ of $\mathscr{S}(A)$, define $\bigcap A_{u}=\operatorname{Ker}\left(A \rightarrow \prod_{u \in U} A / A_{u}\right)$, and $\bigcup A_{u}=\operatorname{Im}\left(\sum_{u \in U} A_{u} \rightarrow A\right)$.

When speaking of the operations defined in Proposition 1.1, we shall use the terms intersection and union, respectively, of the subobjects $A_{u}(u \in U)$. We shall use the notation $\operatorname{Hom}(A, B)$ for the Abelian group of morphisms from $A$ to $B$, where $A$ and $B$ are objects of $\mathscr{C}$, and write $\operatorname{Ext}(A, B)$ for $\operatorname{Ext}^{1}(A, B)$. We say a class $\mathscr{A}$ of objects of $\mathscr{C}$ is closed under group extensions $\left({ }^{2}\right)$ if for any exact sequence

$$
0 \rightarrow A_{1} \rightarrow X \rightarrow A_{2} \rightarrow 0
$$

with $A_{1}, A_{2} \in \mathscr{A}$ it follows that $X \in \mathscr{A}$.

A torsion theory for the Abelian category $\mathscr{C}$ consists of a couple $(\mathscr{T}, \mathscr{F})$ of classes of objects of $\mathscr{C}$ satisfying the following axioms:

(I) $\mathscr{T} \cap \mathscr{F}=\{0\}$.

(II) If $T \rightarrow A \rightarrow 0$ is exact with $T \in \mathscr{T}$ then $A \in \mathscr{T}$.

(III) If $0 \rightarrow A \rightarrow F$ is exact with $F \in \mathscr{F}$ then $A \in \mathscr{F}$.

(IV) For each object $X$ of $\mathscr{C}$ there is an exact sequence

$$
0 \rightarrow T \rightarrow X \rightarrow F \rightarrow 0
$$

with $T \in \mathscr{T}$ and $F \in \mathscr{F}$.

Axiom (IV) will be referred to as the extension axiom. Axioms (I) through (III) may be replaced by the orthogonality axiom.

(V) $\operatorname{Hom}(T, F)=0$ for each $T \in \mathscr{T}, F \in \mathscr{F}$, in the presence of (IV) and the additional assumption that $\mathscr{T}$ and $\mathscr{F}$ each contain with any object all its isomorphic copies in $\mathscr{C}$. If there exists for the class $\mathscr{T}$ a class $\mathscr{F}$ such that $\mathscr{T}$ and $\mathscr{F}$ together satisfy (I)-(IV) we say $\mathscr{T}$ is a torsion class, or T-class. Similarly we define torsion-free class, or F-class.

(1) See Remark (2) at the end of $\$ 5$.

(2) This terminology is used to emphasize the fundamental distinction between this concept and that of "essential extension" below. 
2. Characterizations of torsion theories. If the couple $(\mathscr{T}, \mathscr{F})$ is a torsion theory for $\mathscr{C}$ one verifies in short order that $\mathscr{T}$ and $\mathscr{F}$ are each complete with respect to the orthogonality relation (V). Also, it follows easily from (IV) and (V) that the subobject of $M$ isomorphic to $T$ in (IV) is the least upper bound in $\mathscr{S}(M)$ of the set of subobjects of $M$ which are simultaneously members of $\mathscr{T}$. We have the converse in

THEOREM 2.1. Let $\mathscr{T}$ and $\mathscr{F}$ be complete with respect to the orthogonality relation (V). Then the couple $(\mathscr{T}, \mathscr{F})$ is a torsion theory for $\mathscr{C}$.

Proof. It suffices to verify axiom (IV). To this end we first show that $\mathscr{T}$ is closed under infinite sums (when they exist in $\mathscr{C}$ ) and under group extensions. Let $\left\{T_{u}\right\}_{u \in U}$ be a family of objects of $\mathscr{T}$ whose sum exists in $\mathscr{C}$. If $F$ is an arbitrary member of $\mathscr{F}$, we have

$$
\operatorname{Hom}\left(\sum_{u \in U} T_{u}, F\right) \approx \prod_{u \in U} \operatorname{Hom}\left(T_{u}, F\right)=0
$$

so that $\Sigma_{u \in U} T_{u} \in \mathscr{T}$ by the completeness of $\mathscr{T}$. To see that $\mathscr{T}$ is closed under group extensions, consider the exact sequence

$$
0 \rightarrow T_{1} \rightarrow X \rightarrow T_{2} \rightarrow 0
$$

with $T_{1}, T_{2} \in \mathscr{T}$. For any $F \in \mathscr{F}$, we have the exact sequence of Abelian groups

$$
0 \rightarrow 0=\operatorname{Hom}\left(T_{2}, F\right) \rightarrow \operatorname{Hom}(X, F) \rightarrow \operatorname{Hom}\left(T_{1}, F\right)=0
$$

from which it follows that $X \in \mathscr{T}$.

Now if $M$ is an arbitrary object of $\mathscr{C}$, let $\mathscr{M}$ be the class of subobjects of $M$ which are members of $\mathscr{T}$. By assumption on $\mathscr{C}, \mathscr{M}$ can be indexed by a set, $\mathscr{M}=\left\{M_{u}\right\}_{u \in U}$. Now if $i_{u}: M_{u} \rightarrow M$ is the canonical monomorphism for each subobject $M_{u}$, the image of $\sum i_{u}: \sum M_{u} \rightarrow M$ is equivalent to the least upper bound subobject $M_{t}$ of the $M_{u}(u \in U)$, given by Proposition 1.1, and $M_{t} \in \mathscr{T}$ (by (II) which we assume has been checked at this point). It remains to show that $M / M_{t} \in \mathscr{F}$. Let $T$ be an arbitrary element of $\mathscr{T}$ and suppose that $f: T \rightarrow M / M_{t}$ is given. Then $\operatorname{Im} f$ is of the form $H / M_{t}$ for some subobject $H$ of $M$ containing $M_{t}$ (see, for example, $\left[6\right.$, p. 127]). But then $H / M_{t} \in \mathscr{T}$, and the sequence

$$
0 \rightarrow M_{t} \rightarrow H \rightarrow H / M_{t} \rightarrow 0
$$

is exact with each end object in $\mathscr{T}$, so $H \in \mathscr{T}$ or $H=M_{t}$. Hence $f=0$, completing the proof.

COROLlaRy 2.2. Suppose $\mathscr{T}$ and $\mathscr{F}$ satisfy (V) and are each closed under isomorphic images. Then a necessary and sufficient condition that $\mathscr{T}$ and $\mathscr{F}$ be complete with respect to (V) is that the extension axiom hold. 
The following characterization of torsion classes has been useful for computations.

THEOREM 2.3. The class $\mathscr{T}$ of objects of $\mathscr{C}$ is a torsion class if and only if $\mathscr{T}$ is closed under images, infinite sums, and group extensions. Dually, the class $\mathscr{F}$ is a torsion-free class if and only if $\mathscr{F}$ is closed under kernels, infinite products, and group extensions.

Proof. If $\mathscr{T}$ is a torsion class, we have already observed the stated properties, as in the proof of Theorem 2.1. Conversely, suppose $\mathscr{T}$ has the three closure properties. Denote by $\mathscr{F}$ that class of objects $F$ such that $\operatorname{Hom}(T, F)=0$ for all $T \in \mathscr{T}$. Then $(\mathrm{V})$ is satisfied, and $\mathscr{T}$ and $\mathscr{F}$ are closed under isomorphic images. We now finish the proof by observing that the present hypothesis is just what was verified in the course of proving Theorem 2.1. The proof of the dual statement can be constructed in a dual manner, or better-by passing to the dual category, observing that a torsion theory $(\mathscr{T}, \mathscr{F})$ goes over into the torsion theory $(\mathscr{F}, \mathscr{T})$ for the dual category $\mathscr{C}^{*}$.

We now prove a few results concerning the maximum $\mathscr{T}$-subobject, beginning with the following result, one half of which was observed in the proof of Theorem 2.1.

Proposition 2.4. Let $(\mathscr{T}, \mathscr{F})$ be a torsion theory, and let $M$ be an arbitrary object of $\mathscr{C}$. Then there is a unique largest subobject $M_{t}$ of $M$ which is a member of $\mathscr{T}$. Moreover, $M / M_{t} \in \mathscr{F} . M_{t}$ can be calculated by either of the equalities

(i) $M_{t}=\bigcup\{T \subseteq M \mid T \in \mathscr{T}\}$, or

(ii) $M_{t}=\bigcap\{S \subseteq M \mid M / S \in \mathscr{F}\}$.

Proof. Having observed (i), we prove (ii). Since $M / M_{t} \in \mathscr{F}$, we have one inclusion. On the other hand if $S \subseteq M$ with $M / S \in \mathscr{F}$, then the image of $M_{t}$ under the natural map $M \rightarrow M / S$ is zero, so that $M_{t} \subseteq S$, and the proposition follows.

We see that $M_{t}$ is the largest of all images in $M$ of maps from objects in $\mathscr{T}$ and the smallest of all kernels of maps from $M$ into objects of $\mathscr{F}$.

COROllary 2.5. The correspondence $M \rightarrow M_{t}$ defines a functor $\mathfrak{r}: \mathscr{C} \rightarrow \mathscr{C}$ having the properties:

(i) Given $f: A \rightarrow B, \mathfrak{r}(f): A_{t} \rightarrow B_{t}$ is the restriction of $f$, i.e., $\mathfrak{r}(f)$ is $f$ composed with $A_{t} \rightarrow A$.

(ii) $\mathfrak{r}(A / \mathfrak{r}(A))=0$.

(iii) $\mathfrak{r}^{2}=\mathfrak{r}$.

Proof. Since $\mathscr{T}$ is closed under images, (i) is obvious. Since $M / M_{t} \in \mathscr{F}$, (ii) follows. To see that (iii) holds, simply note that $M_{t}=\mathfrak{r}(M)$ is the largest subobject of $M$ which is a member of $\mathscr{T}$.

REMARKS. A functor $\mathfrak{r}$ satisfying (i) is called a preradical by Maranda [11]. If $\mathfrak{r}$ also satisfies (ii), $\mathfrak{r}$ is termed a radical. A radical $\mathfrak{r}$ is called a torsion radical 
if $\mathfrak{r}$ is left exact, equivalently, if $A \subseteq B$ implies $\mathfrak{r}(A)=A \cap \mathfrak{r}(B)$. One easily verifies that a torsion radical is idempotent, or satisfies (iii). However, the converse is not true. For example, the radical $G \rightarrow G_{D}$ of the category of Abelian groups assigning to each group its maximum divisible subgroup is idempotent but is not a torsion radical. If $G^{1}$ denotes the elements of infinite height of the Abelian group $G$, the functor $G \rightarrow G^{1}$ satisfies (i) and (ii) but not (iii) (one examines the Prüffer group, $[2$, p. 105]). We shall need the following result (see [11, p. 101]):

Proposition 2.6 (MARANDA). If $\mathfrak{r}$ is a preradical of $\mathscr{C}$ and if $A \subseteq \mathfrak{r}(B)$ then $\mathfrak{r}(B / A)=\mathfrak{r}(B) / A$.

COROLLARY 2.7. The class of objects held fixed by a radical $\mathfrak{r}$ is closed under images.

Proof. If $\mathfrak{r}(A)=A$ and $K \subseteq A$ then $\mathfrak{r}(A / K)=\mathfrak{r}(A) / K=A / K$.

We then have the characterization of idempotent radicals in

THEOREM 2.8. Let $\mathscr{C}$ be a subcomplete Abelian category and $\mathfrak{x}$ a subfunctor of the identity. Then $\mathfrak{r}$ is an idempotent radical if and only if the class $\mathscr{T}$ of objects held fixed by $\mathfrak{x}$ is a T-class. Equivalently, $\mathfrak{r}$ is an idempotent radical if and only if the kernel class $\mathscr{F}$ of $\mathfrak{r}$ is an F-class.

Proof. Assume $\mathfrak{r}$ is an idempotent radical. Let $\mathscr{T}$ be the class of objects $A$ such that $\mathfrak{r}(A)=A$, and $\mathscr{F}$ the class of objects $B$ with $\mathfrak{r}(B)=0$. We verify the axioms for a torsion theory. First, (I) is clear. (II) is just Corollary 2.7, and (III) is immediate. Now the exact sequence

$$
0 \rightarrow \mathfrak{r}(A) \rightarrow A \rightarrow A / \mathfrak{r}(A) \rightarrow 0
$$

satisfies the axiom (IV) in view of the hypothesis on $\mathfrak{r}$.

Conversely, define $\mathscr{T}$ and $\mathscr{F}$ as above and suppose $\mathscr{T}$ is a $T$-class. Denoting

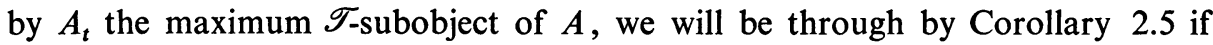
we show that for any object $A, \mathfrak{r}(A)=A_{t}$. But given $A, \mathfrak{r}(A) \subseteq A_{t}$, as $A_{t}$ is the maximum subobject held fixed by $\mathfrak{r}$. On the other hand, $A_{t} \subseteq A$ shows $A_{t}=\mathfrak{r}\left(A_{t}\right) \subseteq \mathfrak{r}(A)$, since $\mathfrak{r}$ is a subfunctor of the identity.

We have noted that $\mathscr{T}$ is closed under subobjects if and only if the functor $A \rightarrow A_{t}$ is left exact. We have the following characterization of this situation when $\mathscr{C}$ has injective envelopes:

THEOREM 2.9. Let $\mathscr{C}$ have injective envelopes and let $(\mathscr{T}, \mathscr{F})$ be a torsion theory for $\mathscr{C}$. Then $\mathscr{T}$ is closed under subobjects if and only if $\mathscr{F}$ is closed under taking injective envelopes.

Proof. Suppose $\mathscr{T}$ is closed under subobjects. We need only show that $\mathscr{F}$ is closed under essential extensions, by a well-known result in [6]. If $F \in \mathscr{F}$, let $E$ be an essential extension of $F$. Then by hypothesis and (III) we have the 
subobject $E_{t} \cap F$ both in $\mathscr{T}$ and in $\mathscr{F}$, so that $E_{t} F=0$, or $E_{t}=0$. Hence $E \in \mathscr{F}$. Conversely, suppose $\mathscr{F}$ is closed under injective envelopes. Then $0 \rightarrow F \rightarrow E(F)$ is exact, where $E(F)$ is an injective envelope of $F$. But $E(F) \in \mathscr{F}$ leads to the exact sequence

$$
0=\operatorname{Hom}(T, E(F)) \rightarrow \operatorname{Hom}(A, E(F)) \rightarrow 0,
$$

from which follows the exact sequence

$$
0 \rightarrow \operatorname{Hom}(A, F) \rightarrow \operatorname{Hom}(A, E(F))=0,
$$

or $A \in \mathscr{T}$.

Whenever the equivalent conditions of 2.7 are valid, $\mathscr{C}$ satisfies the closure axioms of a strongly complete Serre class (see [6], [8], [1], [13], [15], and especially [4] and [16]).

Interesting examples of torsion theories appear naturally in the theory of Abelian groups. For this category we have of course the torsion and torsion-free groups. Another example is the pair of classes consisting of the divisible groups and the reduced groups. In this connection it is of interest to note that under Pontrjagin duality, the $T$-class of ordinary torsion groups goes over into the $F$-class of totally disconnected compact groups [9], from which it is easily deduced that the $T$-class of connected compact groups is the dual of the $F$-class of torsionfree groups, and so on. In order to obtain unlimited examples of torsion theories we now consider some closure operations defined on classes of objects of $\mathscr{C}$.

3. Generation of torsion theories. In this section $\mathscr{C}$ is a fixed Abelian category (subcomplete), and the script letters $\mathscr{A}, \mathscr{B}$ denote classes of objects of $\mathscr{C}$. Then given $\mathscr{A}$, we define operators $L$ and $R$ as follows:

$$
\begin{aligned}
& L(\mathscr{A})=\{B \mid \operatorname{Hom}(B, A)=0 \text { for all } A \in \mathscr{A}\}, \\
& R(\mathscr{A})=\{B \mid \operatorname{Hom}(A, B)=0 \text { for all } A \in \mathscr{A}\} .
\end{aligned}
$$

Proposition 3.1.

Then we have the easily verified result:

(i) $\mathscr{A} \cap L(\mathscr{A})=\{0\}, \mathscr{A} \cap R(\mathscr{A})=\{0\}$.

(ii) $\mathscr{A} \subseteq L R(\mathscr{A}), \mathscr{A} \subseteq R L(\mathscr{A})$.

(iii) If $\mathscr{A} \subseteq \mathscr{B}$, then $L(\mathscr{A}) \supseteq L(\mathscr{B})$ and $R(\mathscr{A}) \supseteq R(\mathscr{B})$.

(iv) $L R L=L, R L R=R$.

(v) $T=L R$ and $F=R L$ are idempotent.

Proposition 3.2. There is a one-one order-reversing correspondence between images of Tand those of $F$, wherein $T(\mathscr{A})$ corresponds to $R T(\mathscr{A})=F R(\mathscr{A})$, and $F(\mathscr{A})$ corresponds to $L F(\mathscr{A})=T L(\mathscr{A})$.

We call a class $\mathscr{A} T$-closed $(F$-closed $)$ if $T(\mathscr{A})=\mathscr{A}(F(\mathscr{A})=\mathscr{A})$. Then we 
note that any image of $L$ is $T$-closed and any image of $R$ is $F$-closed. Then the following proposition is immediate in view of Theorem 2.1. It follows that the notions of $T$-class and $T$-closed class are equivalent.

Proposition 3.3. The following statements are equivalent for the pair $(\mathscr{T}, \mathscr{F})$ of classes of objects of $\mathscr{C}$.

(i) $(\mathscr{T}, \mathscr{F})$ is a torsion theory for $\mathscr{C}$.

(ii) $\mathscr{T}$ is $T$-closed with $R(\mathscr{T})=\mathscr{F}$.

(iii) $\mathscr{F}$ is F-closed with $L(\mathscr{F})=\mathscr{T}$.

(iv) $R(\mathscr{T})=\mathscr{F}$ and $L(\mathscr{F})=\mathscr{T}$.

Proposition 3.4. If $\mathscr{A}_{u}$ are classes of objects of $\mathscr{C}(u \in U)$, then

$$
L\left(\bigcup \mathscr{A}_{u}\right)=L\left(\bigcup R L\left(\mathscr{A}_{u}\right)\right)=\bigcap L\left(\mathscr{A}_{u}\right)
$$

The dual result holds, interchanging $L$ and $R$.

Proof. The proof consists of routine application of Proposition 3.1.

CoRollary 3.5. If the classes $\mathscr{A}_{u}(u \in U)$ are each T-closed (F-closed), then so is their intersection.

Proof. Suppose $T\left(\mathscr{A}_{u}\right)=\mathscr{A}_{u}(u \in U)$. Then we have

$$
T\left(\bigcap \mathscr{A}_{u}\right)=L R\left(\bigcap L R\left(\mathscr{A}_{u}\right)\right)=L R L\left(\bigcup R\left(\mathscr{A}_{u}\right)\right)=L\left(\bigcup R\left(\mathscr{A}_{u}\right)\right)=\bigcap L R\left(\mathscr{A}_{u}\right)=\bigcap \mathscr{A}_{u}
$$

One interchanges $L$ and $R$ to prove the dual result.

COROLlaRy 3.6. Let $\mathscr{T}_{u}(u \in U)$ be T-closed. Then the F-class corresponding to $\bigcap^{\mathscr{T}_{u}}$ is $R\left(\bigcup \mathscr{T}_{u}\right)$. On the other hand, the F-class corresponding to $T\left(\bigcup \mathscr{T}_{u}\right)$ is $\bigcap^{R\left(\mathscr{T}_{u}\right)}$. The dual result holds, interchanging $R$ and $L, T$ and $F$.

4. Specific torsion theories. We now go about the task of constructing several specific torsion theories for $\mathscr{C}$. An object $S$ of $\mathscr{C}$ is called simple if any epimorphism $S \rightarrow E$ is either zero or an isomorphism. In the Abelian category $\mathscr{C}$ this is of course equivalent to the statement that $S$ has no subobjects except 0 and $S$. Now if $(\mathscr{T}, \mathscr{F})$ is a torsion theory for $\mathscr{C}$, then we have either $S \in \mathscr{F}$, or $S \in \mathscr{T}$, according as $S_{t}=0$ or $S_{t}=S$. Hence it is natural to consider the classes $\mathscr{T}_{s}=T(\{S\})$ and $\mathscr{R}_{s}=F(\{S\})$. We shall refer to the former as the class of $S$-primary objects of $\mathscr{C}$ and to the latter as the class of $S$-reduced objects of $\mathscr{C}$. The class $\mathscr{D}_{s}=L F(\{S\})$ is called the class of $S$-divisible objects of $\mathscr{C}$. Finally, the class $\mathscr{F}_{s}=R T(\{S\})$ will be referred to as the class of $S$-free objects of $\mathscr{C}$. If now $\mathscr{S}$ is a representative class of nonisomorphic simple objects of $\mathscr{C}$, then the classes $T(\mathscr{S})=\mathscr{T}_{0}$ and $R T(\mathscr{S})=R\left(\mathscr{T}_{0}\right)=\mathscr{F}_{0}$ will be called the classes of torsion and torsion-free objects of $\mathscr{C}$, respectively. Finally, two further classes will be of interest. $\mathscr{R}=F(\mathscr{S})$ and $\mathscr{D}=L F(\mathscr{S})$ will be called the classes of reduced and divisible objects, respectively. It is easy to check that these classes coincide 
with the classes of these classical names in the category of Abelian groups, or in the category of modules over a Dedekind domain, (not a field), replacing the letter $S$ by the corresponding prime $p$ (our term $S$-free is not classical). By the socle $s(A)$ of an object $A$, we mean the union of zero and all the simple subobjects of $A$. If $S \in \mathscr{S}$, we call the union of the zero subobject and all the simple subobjects of $A$ isomorphic to $S$ the $S$-socle of $A$, and denote it by $A[S]$, following the notation of Abelian group theory. The following proposition is immediate from the above definitions.

Proposition 4.1. $F \in \mathscr{F}_{0}$ is and only if $s(F)=0$, and if $S \in \mathscr{S}, F \in \mathscr{F}_{s}$ if and only if $F[S]=0$.

Proposition 4.2. The T-classes $\mathscr{T}_{s}$ and $\mathscr{T}_{0}$ are closed under subobjects.

Proof. By Theorem 2.8 we need only check that $\mathscr{F}_{s}$ and $\mathscr{F}_{0}$ are closed under essential extensions. So let $F \in \mathscr{F}_{0}$ and let $E$ be an essential extension of $F$. Then if $S$ is a simple subobject of $E, S \cap F=0$, so that $E$ has a zero socle, or $E \in \mathscr{F}_{0}$. The other assertion follows similarly.

Proposition 4.3. If $T \in \mathscr{T}_{0}$, then $T$ is an essential extension of its socle.

Proof. Let $T \in \mathscr{T}_{0}$ and $H \subseteq T$ with $H \cap s(T)=0$. Then $H \in \mathscr{T}_{0}$ by Proposition 4.2 , but $s(H) \subseteq s(T)$. If $s(H)=0$, then $H \in \mathscr{F}_{0}$. Hence $H=0$.

Corollary 4.4. If $A \in \mathscr{T}_{s}$, then $A$ is an essential extension of $A[S]$.

Proof. One notes that every simple subobject of the $S$-primary object $A$ is isomorphic to $S$, so that the assertion follows from Propositions 4.2 and 4.3.

Corollary 4.5. If $S, S^{\prime} \in \mathscr{S}$ with $S \neq S^{\prime}$, then $\mathscr{T}_{s} \cap \mathscr{T}_{s^{\prime}}=\{0\}$.

Corollary 4.6. If $A$ is $S$-primary then $A$ is $S^{\prime}$-divisible for $S^{\prime} \neq S$ in $\mathscr{S}$.

Proof. It is immediate from Corollary 4.5 that $\operatorname{Hom}\left(A, S^{\prime}\right)=0$ for any $S$-primary object $A$.

Corollary 4.7. If $A$ is $S$-primary and $S$-divisible, then $A$ is divisible.

Propositon 4.8. Let $S_{0} \in \mathscr{S}$. Then we have the equality

$$
\mathscr{T}_{s_{0}} \bigcap T\left(\bigcup\left\{\mathscr{T}_{s} \mid S \in \mathscr{S}, S \neq S_{0}\right\}\right)=\{0\} \text {. }
$$

Proof. We have $T\left(\left\{S_{0}\right\}\right) \cap T\left(\bigcup_{s \neq s_{0}} T(\{S\})\right)=T\left(\left\{S_{0}\right\}\right) \cap T\left(\left\{S \mid S \neq S_{0}\right\}\right)$ $=T\left(\left\{S_{0}\right\}\right) \cap L R\left(\left\{S \mid S \neq S_{0}\right\}\right)$, which by Proposition 3.4, is $T\left(\left\{S_{0}\right\}\right) \cap L\left(\bigcap_{s \neq s_{0}}\right.$ $R(\{S\}))=0$. Since for $S \neq S_{0}, T\left(\left\{S_{0}\right\}\right) \subseteq R(\{S\})$ so that $T\left(\left\{S_{0}\right\}\right) \subseteq \bigcap_{s \neq s_{0}} R(\{S\})$ and we are through by (i) of Proposition 3.1. This result generalizes to

Proposition 4.9. Let $\mathscr{A}, \mathscr{B} \subseteq \mathscr{S}$ with $\mathscr{A} \cap \mathscr{B}=\varnothing$. Then $T(\mathscr{A}) \cap T(\mathscr{B})=\{0\}$. Also, $T(\mathscr{A})$ and $T(\mathscr{B})$ are closed under subobjects. 
Proof. $T(\mathscr{A}) \cap T(\mathscr{B})=T(\mathscr{A}) \cap L\left(\bigcap_{s \in \mathscr{B}} R(\{S\})\right)$. But $T(\mathscr{A}) \subseteq R(\{S\})$ for any $S \in \mathscr{B}$, for if $A \in T(\mathscr{A}), A$ is $S$-free for $S \in \mathscr{B}$. Hence $T(\mathscr{A}) \subseteq \bigcap_{s \in \mathscr{B}} R(\{S\})$, and we have $L\left(\bigcap_{s \in \mathscr{B}} R(\{S\})\right) \cap\left[\bigcap_{s \in \mathscr{B}} R(\{S\})\right]=\{0\}$. The second assertion follows by comparison with Proposition 4.2.

If $M$ is any object of $\mathscr{C}$, denote by $M_{t}$ the maximum torsion subobject of $M$ and by $M_{s}$ the maximum $S$-primary subobject of $M$. Then we have a first approximation to a primary decomposition in

THEOREM 4.10. If $M$ is any object of $\mathscr{C}$, then the union of the family of subobjects $M_{s}(S \in \mathscr{S})$ of $M$ is isomorphic to the direct sum $\sum_{s \in \mathscr{S}} M_{s}$, of which $M_{t}$ is an essential extension.

Proof. We need only show that the canonical map

$$
\phi=\Sigma_{i_{M}}: \Sigma_{s \in \mathscr{S}} M_{s} \rightarrow M
$$

is a monomorphism, where $i_{M_{s}}: M_{s} \rightarrow M$ is the canonical injection, for each $S \in \mathscr{S}$. Note that the image of $\phi$ is precisely the union of the subobjects $M_{s}$ in $M_{t} \subseteq M$. Before proceeding, we prove a lemma.

LEMMA $4.11\left(\sum_{s \in \mathscr{S}} M_{s}\right)_{s^{\prime}}=M_{s^{\prime}}$ for any $S^{\prime} \in \mathscr{S}$.

Proof. Clearly $M_{s^{\prime}} \subseteq\left(\Sigma_{s \in \mathscr{S}} M_{s}\right)_{s^{\prime}}$. For the opposite inclusion we need $\left(\sum_{s \in \mathscr{S}} M_{s}\right) / M_{s^{\prime}} \in \mathscr{F}_{s^{\prime}}\left(=R T\left(\left\{S^{\prime}\right\}\right)\right.$, by Proposition 2.4, (ii)). But this factor object is naturally isomorphic to the sum $\sum_{s \neq s^{\prime}} M_{s} \in \mathscr{F}_{s^{\prime}}$. We now return to the proof of the theorem. Let $E$ be arbitrary in $\mathscr{C}$ and $f: E \rightarrow \Sigma_{s \in \mathscr{S}} M_{s}$ such that $\phi f=0$. Then $(\phi(\operatorname{Im} f))_{s}=0$ for each $S \in \mathscr{S}$. Let $i_{s}$ be the injection furnished by the sum for each $S \in \mathscr{S}$. Then $\phi i_{s}=i_{M}$ is a monomorphism for each $S \in \mathscr{S}$ such that. $M_{s} \neq 0$. We have $(\operatorname{Im} f)_{s} \subseteq M_{s}$, by the lemma and hence $\phi\left((\operatorname{Im} f)_{s}\right) \subseteq(\phi(\operatorname{Im} f))_{s}=0$, which implies that $\phi i_{s}\left((\operatorname{Im} f)_{s}\right)=i_{M_{s}}\left((\operatorname{Im} f)_{s}\right)=(\operatorname{Im} f)_{s}=0$ for each $S \in \mathscr{S}$. Hence $\operatorname{Im} f$ is a torsion object with zero socle, or is zero. Hence $f=0$.

Remarks. The above result cannot be improved in some Abelian categories Walker [15] gives an example of an Abelian category having nonisomorphic simple objects $S_{1}$ and $S_{2}$ with $\operatorname{Ext}\left(S_{1}, S_{2}\right) \neq 0$. Hence there is an exact sequence $0 \rightarrow S_{2} \rightarrow X \rightarrow S_{1} \rightarrow 0$ which is nonsplitting, yet $X$ is torsion with its $S_{2}$-primary part isomorphic to $S_{2}$ and its $S_{1}$-primary part zero.

If the direct sum of injectives is injective and the classes $\mathscr{T}_{s}$ are closed under taking injective envelopes for each $S \in \mathscr{S}$, we can deduce rather easily a primary decomposition for the torsion injectives by methods used in [4], noticing that the direct sum of the injective envelopes of the simple objects in the socle of a torsion injective $Q$ is a torsion essential extension of $\mathrm{s}(Q)$, and hence that the sum is $Q$ itself. Note also that in this situation, each injective object of $\mathscr{C}$ splits into its torsion and torsion-free parts. Gabriel [4] has obtained a further decomposition of the torsion-free part into indecomposable injectives. 
Concerning the phenomenon that the $S$-primary objects are closed under essential extensions, we have

Proposition 4.12. The following statements are equivalent when $\mathscr{C}$ has injective envelopes:

(i) $\mathscr{T}_{s}$ is closed under essential extensions for each $S \in \mathscr{S}$.

(ii) Any $S$-primary object can be imbedded in an $S$-primary injective object.

(iii) Any injective object $Q$ decomposes as $Q=Q_{s} \oplus F$, where $F$ is unique up to isomorphism.

(iv) If $A[S]$ is essential in $A$ then $A$ is $S$-primary.

(v) For any object $A, A_{s}$ is the unique maximal essential extension in $A$ of the S-socle $A[S]$.

Proof. That (i) implies (ii) follows from elementary properties of essential extensions. Also, if (ii) holds, the injective envelope of the $S$-socle of $Q$ is also $S$-primary, and is a summand of $Q$. Assuming (iii), let $A$ be an essential extension of $A[S]$. Then $A$ is contained in the $S$-primary injective envelope of $A[S]$. Now assume (iv), and let $A$ be any object of $\mathscr{C}$. The union in $A$ of all essential extensions of the $S$-socle of $A$ is $S$-primary, being a direct limit of $S$-primary subobjects, and is thus an essential extension of its $S$-socle $A[S]$, which has the required property by its construction. To see that (v) implies (i), let $A$ be $S_{0}$-primary. If $E$ is an essential extension of $A, E$ is an essential extension of $S(A)=A\left[S_{0}\right]$ in $E$, so that $E$ is $S_{0}$-primary. With a similar proof, we have the corresponding result:

Proposition 4.13. The following are equivalent when $\mathscr{C}$ has injective envelopes:

(i) $\mathscr{T}_{0}$ is closed under essential extensions.

(ii) Any torsion object can be imbedded in a torsion injective.

(iii) Any injective object $A$ decomposes as $A=A_{t} \oplus F$, where $A_{t}$ is the torsion part of $A$ and $F$ is unique up to isomorphism and has no socle.

(iv) If $A$ is an essential extension of $s(A)$, then $A$ is torsion.

(v) For any object $A, A_{t}$ is the unique maximal essential extension in $A$ of the socle $s(A)$.

Matlis in [12] has shown that for modules over a Noetherian commutative ring $R$ with unit a primary decomposition holds for all modules with maximal orders. For a given maximal ideal $\mathscr{M}$ of $R$, a module $A$ is said to be $\mathscr{M}$-primary if for each $x \in A, \mathscr{M}^{n} x=0$ for some $n>0$. A module $A$ is said to have maximal orders if for each $x \in A$, any prime ideal of $R$ containing $(0: x)$ is maximal. One verifies easily from results in [12] that in the Noetherian case, the $\mathscr{M}$-primary modules coincide with the $S$-primary modules in our sense, where $S=R / \mathscr{M}$, and that the modules with maximal orders coincide with our class $\mathscr{T}_{0}$. For integral 
domains $R$ such that any nonzero prime ideal is maximal, e.g., Dedekind domains, the class of modules with maximal orders coincides with the usual order ideal definition of torsion. We shall give now an example of a commutative ring $R$ with unit (non-Noetherian) such that the conditions of Proposition 4.13 are not satisfied for its modules, but every $R$-module has maximal orders. It will be apparent that not every $R$-module decomposes in the sense of [12]. Let $R$ be the product of countably many nonisomorphic fields, $R=\prod F_{i}\left(F_{i}\right.$ the integers modulo $p_{i}$, where $p_{i}$ is the $i$ th prime, will do).

LEMMA: 4.14. Each prime ideal of $R$ is maximal.

Proof. If $P$ is a prime ideal of $R$ and $x \in R, x \notin P$, let $y x=0$. Then $y x \in P$ so $y \in P$. In particular, consider an element $e_{x}$ such that $\left(e_{x}\right)_{i}=0$ when $(x)_{i} \neq 0,\left(e_{x}\right)_{i}=1$ otherwise. Then $e_{x} \in P$, and since $F_{i}$ is a field, there is an element $r \in R$, such that $(r x)_{i}=0$ or 1 for cach $i$ and also $r x+e_{x}=e$, where $e$ is the unit of $R$. Thus $P+R x=R$, and it follows that $P$ is maximal.

LEMmA 4.15. The $R$-module $\prod F_{i} / \sum F_{i}$ has zero socle.

Proof. Let $\Sigma$ denote the sum $\Sigma F_{i}$, and let $\bar{x}=x+\sum$ be a nonzero element of the quotient. Let $I=\left\{n_{k}\right\}_{k=1}^{\infty}$ be that infinite subset of the positive integers such that $(x)_{n_{k}} \neq 0, k=1,2, \cdots$. One easily verifies that $A=(0: \bar{x})$ is just the set of $t \in R$ such that $(t)_{i}=0$ for all but finitely many $i \in I$. We show that the ideal $A$ is not maximal. Define $(y)_{j}=1$, if $j=n_{2 k}$ in $I,(y)_{j}=0$ otherwise. Then $y \notin A$, yet there is no $r \in R, a \in A$ such that $r y+a=e$. For no matter which $r$ is chosen, $(r y+a)_{j}=0$ for infinitely many $j$ of the form $j=n_{2 k+1} \in I$, which concludes the proof.

Remarks. Lemma 4.14 shows that each $R$-module has maximal orders, although it is apparent that $R$ does not decompose into a direct sum of its $\mathscr{M}$-primary submodules. Indeed, $R$ is an essential extension of $\Sigma$, but since $R / \sum$ is a nonzero torsion-free module, it is clear that the socle is contained in $\Sigma$, which thus coincides with the socle.

5. The primary decomposition. In this section $\mathscr{C}$ is a subcomplete Abelian category with injective envelopes. As before, $\mathscr{S}$ denotes a representative class of nonisomorphic simple objects of $\mathscr{C}$, and we use the words "torsion" and " $S$-primary" as in the previous section. We are concerned with the primary decomposition:

For any torsion object $A, A=\sum_{s \in \mathscr{S}} A_{s}$.

Lemma 5.1. Assume (PD) holds. If $B$ is a torsion object and $A \subseteq B$, then the sequence

$$
0 \rightarrow A_{s} \stackrel{i \mid}{\rightarrow} B_{s} \stackrel{\pi \mid}{\rightarrow}(B / A)_{s} \rightarrow 0
$$


is exact, where $i \mid$ and $\pi \mid$ denote the restrictions of the inclusion $i: A \rightarrow B$ and its cokernel $\pi$, respectively.

Proof. The sequence (E) is left exact by previous work, and to show that it is right exact, it suffices to show that every $S$-primary subobject of $B / A$ is an image under $\pi \mid$ of some $S$-primary subobject of $B$. To this end, let $H / A$ be a subobject of $(B / A)_{s}$. Then $\pi(H)=H / A$, but in view of the primary decomposition of $H$ and Proposition 4.9, it is clear that $\pi\left(\sum_{s^{\prime} \neq s} H_{s^{\prime}}\right)=0$, so that $\pi(H)=\pi\left(H_{s}\right) \cup \pi\left(\sum_{s^{\prime} \neq s} H_{s^{\prime}}\right)=\pi\left(H_{s}\right) \cup 0=\pi\left(H_{s}\right)$, and the proof is complete.

Proposition 5.2. Suppose that for any exact sequence

$$
0 \rightarrow A \rightarrow B \rightarrow C \rightarrow 0
$$

of torsion objects, the associated sequence

$$
0 \rightarrow A_{s} \rightarrow B_{s} \rightarrow C_{s} \rightarrow 0
$$

is exact, for each $S \in \mathscr{S}$. Then a torsion essential extension of an S-primary object is S-primary.

Proof. Suppose $A$ is $S$-primary, and $E$ a torsion essential extension of $A$. Then consider the exact sequence

$$
0 \rightarrow E_{s} \rightarrow E \rightarrow E / E_{s} \rightarrow 0 .
$$

If $S_{1} \neq S \in \mathscr{S}$, the sequence

$$
0 \rightarrow 0=\left(E_{s}\right)_{s_{1}} \rightarrow 0=E_{s_{1}} \rightarrow\left(E / E_{s}\right)_{s_{1}} \rightarrow 0
$$

is exact, or $\left(E / E_{s}\right)_{s_{1}}=0$. We already know $\left(E / E_{s}\right)_{s}=0$, so that the object $E / E_{s}$ is torsion and has zero socle, and is therefore zero, as desired.

We now turn to the main result of this section.

THEOREM 5.3. (PD) holds if and only if for each $S \in \mathscr{S}$, the functor $A \rightarrow A_{s}$ is exact on the full subcategory $\mathscr{T}_{0}$.

Proof. Assuming (PD), the necessity of the condition is just 5.1. To prove the converse it suffices to show that the class $\mathscr{T}_{1}$ of objects $A$ of the form $A=\sum_{s \in \mathscr{S}} A_{s}$ is a torsion class, for it clearly contains the simple objects and thus would contain $\mathscr{T}_{0}$, the minimum such class. The class $\mathscr{T}_{1}$ is obviously closed under images and direct sums, hence it suffices to show that $\mathscr{T}_{1}$ is closed under group extensions, by Theorem 2.3. But an exact sequence of the form

$$
0 \rightarrow \Sigma_{s \in \mathscr{S}} A_{s} \rightarrow X \rightarrow \Sigma_{s \in \mathscr{S}} B_{s} \rightarrow 0
$$

yields $0 \rightarrow A_{s} \rightarrow X_{s} \rightarrow B_{s} \rightarrow 0$ exact for each $s \in \mathscr{S}$ by Lemma 4.11 so that $\sum_{s \in \mathscr{S}} X_{s} / \Sigma_{s \in \mathscr{S}} A_{s}=\sum_{s \in \mathscr{S}} B_{s}$ and $X=\sum_{s \in \mathscr{S}} X_{s}$ follows. This version of the proof was discovered after the manuscript had been sent for publication. The referee found 
the following elegant proof of the converse: If $T$ is a torsion object, let $A=\Sigma_{s \in \mathscr{S}} T_{s}$; then the sequence $0 \rightarrow A \rightarrow T \rightarrow T / A \rightarrow 0$ is exact, and hence $0 \rightarrow A_{s} \rightarrow T_{s} \rightarrow(T / A)_{s} \rightarrow 0$ is exact for all $s \in \mathscr{S}$. But $A_{s}=T_{s}$ by Lemma 4.11 and so $(T / A)_{s}=0$ for all $s \in \mathscr{S}$. Thus $T / A$ is torsion-free. Since $T$ is torsion, so is $T / A$, and so $T=A$.

REMARKS. (1) Some investigations on the question of which module categories satisfy the hypothesis of Theorem 5.3 will appear in a separate publication.

(2) We have chosen to work with a subcomplete Abelian category in $\S \S 1$ and 2 mainly to avoid the Grothendieck A.B.5 axiom which would be involved in a Zorn lemma proof of the existence of a maximum torsion subobject. It is well known that the A.B.5 axiom introduces a certain amount of nonduality which we have avoided as much as possible. Actually for our purposes all that is needed is that the subobject lattices $\mathscr{S}(A)$ are complete both in $\mathscr{C}$ and in $\mathscr{C}^{*}$. For Theorem 2.7 we have assumed injective envelopes rather than to assume A.B.5 along with the other hypotheses used in [6] to establish the existence of injective envelopes. In this connection S. A. Amitsur has pointed out (oral communication) that by a Zorn lemma argument one can show that if $(\mathscr{T}, \mathscr{F})$ is a torsion theory then $\mathscr{T}$ is closed under subobjects if and only if $\mathscr{F}$ is closed under essential extensions - of course the A.B.5 axiom is used.

\section{REFERENCES}

1. S. Balcerzyk, On classes of Abelian groups, Fund. Math. 51 (1962), 149-178.

2. L. Fuchs, Abelian groups, Publ. House Hungarian Acad. Sci., Budapest, 1958.

3. P. Freyd, Abelian categories, Mimeographed Notes, Columbia Univ., New York, 1962.

4. P. Gabriel, Des catégories Abéliennes, Bull. Soc. Math. France 90 (1962), 323-448.

5. E. Gentile, Singular submodule and injective hull, Indag. Math. 24 (1962), 426-433.

6. A. Grothendieck, Sur quelques points d'algèbre homologique, Tôhoku Math. J. (2) 9 (1957), 119-221.

7. A. Hattori, $A$ foundation of torsion theory for modules over general rings, Nagoya Math. J. 17 (1960), 147-158.

8. S. T. Hu, Homotopy theory, Academic Press, New York, 1959.

9. I. Kaplansky, Infinite Abelian groups, Michigan Univ. Press, Ann. Arbor, Mich., 1954.

10. L. Levy, Torsion-free and divisible modules over Noetherian integral domains, Canad. J. Math. 15 (1963), 132-151.

11. J.-M. Maranda, Injective structures, Trans. Amer. Math. Soc. 110 (1964), 98-135.

12. E. Matlis, Modules with descending chain condition, Trans. Amer. Math. Soc. 97 (1960), 495-508.

13. E. J. Peake, Jr., Serre classes of Abelian groups, Doctoral dissertation, New Mexico State Univ., University Park, N. M., 1963.

14. C. Walker, Relative homological algebra and abelian groups (to appear).

15. E. A. Walker, Quotient categories and quasi-isomorphisms of Abelian groups, Proceedings of the Colloquium on Abelian Groups, Tihany (Hungary), 1963, 147-162.

16. E. A. Walker and C. Walker, Quotient categories and rings of quotients (to appear).

\section{UNIVERSITY OF NEBRASKA, LINCOLN, NEBRASKA}

